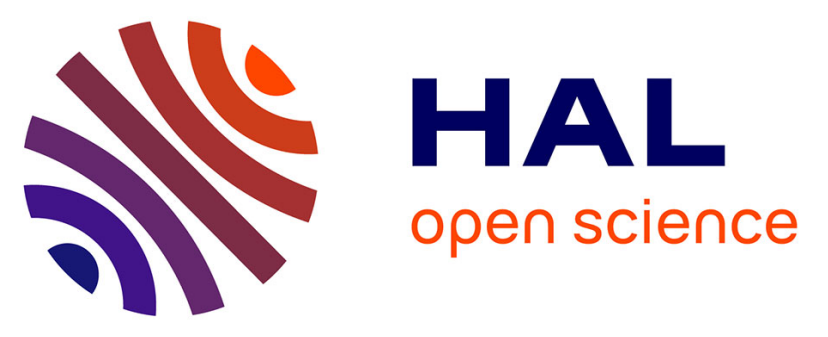

\title{
Maternal obesity leads to long-term altered levels of plasma ceramides in the offspring as revealed by a longitudinal lipidomic study in children
}

\author{
Luis Felipe León-Aguilar, Mikael Croyal, Véronique Ferchaud-Roucher,
} Fengyang Huang, Laurence Marchat, Albino Barraza-Villarreal, Isabelle Romieu, Usha Ramakrishnan, Michel Krempf, Khadija Ouguerram, et al.

\section{To cite this version:}

Luis Felipe León-Aguilar, Mikael Croyal, Véronique Ferchaud-Roucher, Fengyang Huang, Laurence Marchat, et al.. Maternal obesity leads to long-term altered levels of plasma ceramides in the offspring as revealed by a longitudinal lipidomic study in children. International Journal of Obesity, 2019, 43 (6), pp.1231-1243. 10.1038/s41366-018-0291-y . hal-02429513

\section{HAL Id: hal-02429513 \\ https://hal.science/hal-02429513}

Submitted on 6 Jan 2020

HAL is a multi-disciplinary open access archive for the deposit and dissemination of scientific research documents, whether they are published or not. The documents may come from teaching and research institutions in France or abroad, or from public or private research centers.
L'archive ouverte pluridisciplinaire HAL, est destinée au dépôt et à la diffusion de documents scientifiques de niveau recherche, publiés ou non, émanant des établissements d'enseignement et de recherche français ou étrangers, des laboratoires publics ou privés. 

ceramides in the offspring as revealed by a longitudinal lipidomic study in children

\author{
Luis Felipe León Aguilar ${ }^{1,3} \cdot$ Mikael Croyal $^{1} \cdot$ Véronique Ferchaud-Roucher ${ }^{1} \cdot$ Fengyang Huang $^{2}$. \\ Laurence A. Marchat ${ }^{3}$ - Albino Barraza-Villarreal ${ }^{4}$ - Isabelle Romieu ${ }^{4,5}$ - Usha Ramakrishnan ${ }^{5}$. Michel Krempf ${ }^{1}$. \\ Khadija Ouguerram $^{1} \cdot$ Rosalio Mercado-Camargo ${ }^{6} \cdot$ Francisco Bolaños-Jiménez $^{1}$
}

Received: 15 March 2018 / Revised: 21 November 2018 / Accepted: 30 November 2018

(c) Springer Nature Limited 2018

\begin{abstract}
Background/objectives Maternal obesity is associated with increased risk of obesity and other symptoms of the metabolic syndrome in the offspring. Nevertheless, the molecular mechanisms and cellular factors underlying this enhanced disease susceptibility remain to be determined. Here, we aimed at identifying changes in plasma lipids in offspring of obese mothers that might underpin, and serve as early biomarkers of, their enhanced metabolic disease risk.

Subjects/methods We performed a longitudinal lipidomic profiling in plasma samples from normal weight, overweight, and obese pregnant women and their children that participated in the Prenatal Omega-3 Fatty Acid Supplementation, Growth, and Development trial conducted in Mexico. At recruitment women were aged between 18 and 35 years and in week 18-22 of pregnancy. Blood samples were collected at term delivery by venipuncture from mothers and from the umbilical cord of their newborns and from the same infants at 4 years old under non-fasting conditions. Lipidomic profiling was done using ultra-performance liquid chromatography high-resolution mass spectrometry.

Results Analysis of the lipidomic data showed that overweight and obese mothers exhibited a significant reduction in the total abundance of ceramides (Cer) in plasma, mainly of Cer (d18:1/20:0), Cer (d18:1/22:0), Cer (d18:1/23:0), and Cer (d18:1/24:0), compared with mothers of normal body weight. This reduction was confirmed by the direct quantification of these and other ceramide species. Similar quantitative differences in the plasma concentration of Cer (d18:1/22:0), Cer (d18:1/23:0), and Cer (d18:1/24:0), were also found between 4-year-old children of overweight and obese mothers compared with children of mothers of normal body weight. Noteworthy, children exhibited equal daily amounts of energy and food intake independently of the BMI of their mothers.

Conclusions Maternal obesity results in long-lasting changes in plasma ceramides in the offspring suggesting that these lipids might be used as early predictors of metabolic disease risk due to maternal obesity.
\end{abstract}

\title{
Introduction
}

Supplementary information The online version of this article (https:// doi.org/10.1038/s41366-018-0291-y) contains supplementary material, which is available to authorized users.

Francisco Bolaños-Jiménez

Francisco.Bolanos@univ-nantes.fr

1 INRA, UMR1280 Physiologie des Adaptations Nutritionnelles, Université de Nantes, Nantes Atlantique Université, Nantes, France

2 Laboratorio de Farmacología y Toxicología, Hospital Infantil de México Federico Gómez, México City, Mexico

3 Programa de Biotecnología, Escuela Nacional de Medicina y
Obesity is a chronic pathological condition characterized by the excessive accumulation of adipose tissue often complicated by one of the core components of the metabolic

Homeopatía, Instituto Politécnico Nacional, México City, Mexico

4 Population Health Research Center, National Institute of Public Health, Cuernavaca, Mexico

5 Hubert Department of Global Health, Rollins School of Public Health, Emory University, Atlanta, GA, USA

6 Facultad de Químico-Farmacobiología, Universidad Michoacana de San Nicolás de Hidalgo, Morelia, Michoacán, Mexico 
syndrome. Obesity is also a primary risk factor for type 2 diabetes and coronary artery disease and has become a global public health burden affecting all segments of the population [1]. According to the World Health Organization, 41 million children under the age of 5 years are overweight or obese, and 650 million adults are obese, among which $15 \%$ are women [2]. The prevalence of obesity in pregnant women is as high as 30\% [3, 4] and $\sim 40 \%$ of women gain an excessive amount of weight during pregnancy in western countries [3, 5]. This is of special concern because maternal obesity or excessive body weight gain during pregnancy leads to an increased risk of developing metabolic syndrome and cardiovascular diseases in the offspring, via a developmental programming process not only in the first but also in the second generation, thus initiating a vicious cycle to perpetuate chronic degenerative diseases [6-9]. However, there is little information about the mechanistic link between maternal obesity and the longterm adverse metabolic outcomes in the offspring. In addition, it remains to be clearly established whether the increased disease risk exhibited by children born to obese mothers is due to their exposure to an imbalanced nutritional environment during fetal development or results from genetic factors and/or deleterious familial life style habits.

Lipotoxicity is a hallmark of several of the pathological conditions associated with obesity as heart attacks, strokes peripheral vascular diseases [10], and non-alcoholic fatty liver disease $[11,12]$. The accumulation of fatty acids in skeletal muscle leads also to inhibition of insulin receptor signaling, increased production of inflammatory and stress factors, as well as impaired mitochondrial $\beta$-oxidation [13, 14]. Overall, these lipid-mediated changes contribute to diabetes and other chronic degenerative diseases associated with obesity.

Given the key role of altered lipid metabolism in the etiology of obesity-related disorders, the quantification of blood lipids is routinely used in the clinic to estimate metabolic and cardiovascular disease risk, and has also been employed to determine the susceptibility of children born to obese mothers to developing the metabolic syndrome [15, 16]. However, these latter analysis have been restricted to a small number of circulating lipids, namely triacylglycerol (TG), total cholesterol and low-density lipoprotein cholesterol and high-density lipoprotein cholesterol (LDL-C, HDL-C), thus providing a very narrow overview of the alterations occurring in the circulating levels of the many hundreds of individual lipid species that constitute the blood lipidome. Actually, increasing evidence suggests that sphingolipids and other less abundant circulating bioactive lipids play an important role in the etiology of several obesity-related diseases $[17,18]$. Here, we performed a longitudinal lipidomic analysis in plasma samples of children born to lean, overweight, or obese mothers in order to identify changes in lipid profile that might underpin, and serve as early biomarkers of, the disease risk resulting from maternal obesity.

\section{Materials and methods}

\section{Study population and sample collection}

This study was conducted in plasma samples that were obtained from pregnant women and their children who gave their informed consent to participate in the Prenatal Omega3 Fatty Acid Supplementation, Growth, and Development (POSGRAD) trial registered at the Instituto Nacional de Salud Pública (INSP) in Mexico (\#CI-011) and at clinicaltrials.gov (NCT00646360). The design of the trial has been described in detail elsewhere [19]. Eligible women were aged between 18 and 35 years old and between 18 and 22 weeks of pregnancy. Blood samples were collected from mothers and from the umbilical cord blood of the babies by venipuncture at term delivery, as well as from the same infants at 4 years old under non-fasting conditions. Since the original objective of the study was to evaluate the impact of maternal docosahexaenoic acid (DHA) supplementation during pregnancy on the offspring's cognitive development, maternal blood samples during pregnancy were not collected. After collection, blood samples were placed into a tube containing EDTA, and blood cells were separated from plasma which was frozen and stored at $-70{ }^{\circ} \mathrm{C}$ until analysis. The original study included 973 women who received capsules containing either DHA $(n=$ $485)$ or olive oil as placebo $(n=488)$ and that delivered 973 live infants. Among these children, 524 were followed-up during 4 years of whom 341 were excluded because of the lack of complete information or enough amount of plasma sample to give a final number of 91 children in the DHA group and 92 in the placebo group. (Supplemental Fig. 1). For this study, we used 47 mother-infant dyads from the placebo group with each dyad having three plasma samples corresponding to the maternal and new-born samples taken at delivery and the samples of the children at 4 years. Mothers were categorized by standard convention as normal body weight $(18.5 \leq \mathrm{BMI}<25)$, overweight $(25 \leq \mathrm{BMI}<$ $30)$, or obese (BMI $\geq 30)$.

\section{Dietary assessment}

The daily intake of energy of the offspring at 4 years was estimated using a food frequency questionnaire of 108 food items adapted to the Mexican population as described by Hernández-Avila et al. [20] and Romieu et al. [21]. Daily nutrient intakes were obtained by multiplying the portion size of each food item reported in the questionnaire by the 
weight corresponding to its frequency of consumption and by its nutrient value obtained from the food composition tables of the US Department of Agriculture (https://ndb.nal. usda.gov/ndb/) or other sources for specific Mexican foods and portion sizes (http://www.myfitnesspal.com/es/food/ca lorie-chart-nutrition-facts). Total daily calorie intake was calculated by adding the results corresponding to all food items. We also calculated the daily intake of each group of food and its contribution to total energy intake.

\section{Metabolite determinations}

Plasma from children was assayed for insulin, glucose, TG, total cholesterol, HDL-C, LDL-C, aspartate aminotransferase (AST), alanine aminotransferase (ALT), gammaglutamyl-transferase (GGT), creatinine, and uric acid using commercially available reagents as previously described [22].

\section{Lipidomic analysis}

The lipidomic analysis was performed by ultra-performance liquid chromatography-tandem mass spectrometry (UPLCMS/MS) as described in our previous publications [23, 24]. A mixture of seven endogenous standards (Supplemental Table S1) was added to $25 \mu \mathrm{L}$ of each plasma sample and to $25 \mu \mathrm{L}$ of a quality control pool (QC) that was constituted by mixing an equal amount of each plasma sample. Lipids were extracted by the Bligh and Dyer method [25]. Lipid separation was achieved on an Acquity ${ }^{\circledR}$ H-Class UPLC system (Waters Corporation, Milford, USA) equipped of an Acquity ${ }^{\circledast}$ charge surface hybrid $\left(\mathrm{CSH} \mathrm{C} \mathrm{C}_{18}\right)$ column $(2.1$ $\mathrm{mm} \times 100 \mathrm{~mm}, 1.7 \mu \mathrm{m}$; Waters, France) held at $55^{\circ} \mathrm{C}$. Detection of lipids was performed by a Synapt G2 HDMS, Q-TOF mass spectrometer equipped with a Z-Spray interface for electrospray ionization (ESI) (Waters Corporation). The resolution mode was used to scan ions with mass-tocharge ratios $(\mathrm{m} / \mathrm{z})$ from 50 to 1200 at a mass resolution of 25,000 full width half maximum (FWHM) for both positive $(\mathrm{ESI}+)$ and negative $(\mathrm{ESI}-)$ ionization modes. Data were collected in the centroid mode at a rate of four spectra per second. A solution of leucine enkephalin $(2 \mu \mathrm{g} / \mathrm{mL}$ in an acetonitrile:water), containing $0.1 \%$ of formic acid, was infused at a constant flow of $10 \mu \mathrm{L} / \mathrm{min}$ in the lockspray channel to correct the measured $\mathrm{m} / \mathrm{z}$ ratio during experiments. Mass correction was applied throughout the batch at $\mathrm{m} / z$ 556.2771 and 554.2616 for ESI+ and ESI-, respectively. To evaluate the performance of the analytical system and control for inter run changes in sensitivity of the mass spectrometer, QC samples were included in each run. All samples were analyzed in a random order within a period no longer than one week between the first and the last run.
Data acquisition and processing of mass spectrometry data, including peak detection, integration, alignment, and normalization, were achieved using MassLynx ${ }^{\circledR}$ and MakerLynx ${ }^{\oplus}$ software (version 4.1, Waters Corporation, Milford, MA, USA). Lipid markers were selected from the detected features using an in-house lipidomic database of 180 lipid species built by the use of lipid standards, the exact mass measured, the elemental compositions with a mass error below $5 \mathrm{ppm}$, the retention times, and the fragmentation patterns as described in other studies [26, 27]. The relative standard deviation (RSD, \%) was calculated for peak areas to highlight the repeatability of the analytical process. Finally, selected lipid markers having a RSD value below $30 \%$ were retained for multivariate analysis (30).

\section{Quantification of plasma ceramides}

We used the method described by Croyal et al. [23], to quantify different species of ceramides. A pool of standard solutions, including 10 species of ceramides (Cer), was prepared and then serially diluted in methanol (Sigma Aldrich, Saint-Quentin Fallavier, France), to obtain seven standard solutions at $1,5,10,50,100,250$, and $500 \mathrm{nM}$. Standard solutions and plasma samples $(25 \mu \mathrm{L})$, were extracted as described in the previous section after the addition of Cer (d18:1/17:0) as internal standard. UPLCMS/MS analyses were performed on a Xevo ${ }^{\circ}$ triple-quadrupole mass spectrometer with an ESI interface and an Acquity H-Class ${ }^{\bullet}$ UPLC $^{\mathrm{TM}}$ device (Waters Corporation). Data acquisition and analyses were performed with MassLynx ${ }^{\circ}$ and TargetLynx ${ }^{\circ}$ software, respectively (version 4.1, Waters Corporation, Milford, MA, USA). Compounds were separated on an Acquity ${ }^{\oplus} \mathrm{BEH} \mathrm{C}_{18}$ column $(2.1 \times 50$ $\mathrm{mm}, 1.7 \mu \mathrm{m}$, Waters). Ceramides and internal standards were detected by the mass spectrometer with the ESI interface operating in the positive ion mode. The multiple reaction mode (MRM) was applied as described in Supplemental Table S2. Chromatographic peak area ratios between ceramides and their internal standard constituted the detector responses. Standard solutions were used to plot calibration curves and a linear regression model (1/x weighting) was used for quantification. The intra-assay and inter-assay variabilities of the analytical method were assessed over three distinct experiments (four concentration levels, six replicates by level), and were below $13.6 \%$. Recoveries were assessed with internal standard and were $>91 \%$.

\section{Statistical analysis}

UPLC-MS/MS spectral data were first normalized by dividing the intensity of each specific signal, corresponding to a putative marker, by the sum of the intensities of all 
Table 1 Clinical characteristics of mothers and their offspring at birth and at 4 years

Variable Maternal body weight characteristics

One-way ANOVA $p$-value

Normal weight $(n=18) \quad$ Overweight $(n=18) \quad$ Obese $(n=11)$

Maternal characteristics

Height (m)

BMI $\left(\mathrm{kg} / \mathrm{m}^{2}\right)$

$1.56(1.54-1.58)$

$21.86(21.24-22.48)$

Pregnancy weight gain $(\mathrm{kg})$

Age (years)

$13.78(12.83-14.72)$

$24.59(22.43-26.75)$

Time of delivery

13h18 (9h37-16h18)

Infant characteristics at birth

Gestational age (weeks)

Weight

38.61 (37.68-39.54)

Boys

$3.38(3.15-3.62)$

Girls

$1.53(1.50-1.56)$
$26.20(25.93-26.46)$
$14.17(13.17-15.16)$
$25.56(24.36-28.37)$
$13 h 50(11 \mathrm{~h} 10-16 \mathrm{~h} 40)$
$39.40(38.88-39.91)$
$3.40(3.07-3.74)$
9
9

8
Child characteristics and metabolite profile in serum at 4 years

Body weight $(\mathrm{kg})$
Height $(\mathrm{cm})$
BMI $z$-score
Fasting length $(\mathrm{h})$
Insulin (mUI/mL)
Glucose (mg/dL)
Creatinine (mg/dL)
Uric acid (mg/dL)
Triglycerides (mg/dL)
Cholesterol (mg/dL)
HDL-C (mg/dL)
LDL-C (mg/dL)
AST (UI/L)
ALT (UI/L)
GGT (UI/L)

$\begin{array}{cc}15.63(14.90-16.37) & 17.41(15.55-19.27) \\ 101.8(99.75-103.90) & 103.30(100.30-106.20) \\ -0.17(-0.54 \text { to } 0.19) & 0.49(-0.14 \text { to } 1.12) \\ 3.6(3.0-4.13) & 2.79(2.32-3.26) \\ 8.03(4.12-11.95) & 5.60(3.87-7.34) \\ 93.61(89.02-98.20) & 92.11(88.12-96.10) \\ 0.33(0.29-0.37) & 0.36(0.32-0.40) \\ 4.01(3.67-4.37) & 3.98(3.64-4.34) \\ 95.17(74.33-116.00) & 123.50(93.19-153.80) \\ 155.50(143.30-167.70) & 176.60(164.10-189.30)^{*} \\ 53.28(47.79-58.77) & 54.67(50.23-59.10) \\ 83.22(71.89-94.56) & 99.06(87.83-110.30) \\ 34.50(33.17-35.83) & 38.67(36.02-41.31)^{*} \\ 16.17(14.34-18.00) & 21.06(17.44-24.67) \\ 9.66(8.49-10.85) & 10.94(9.54-12.35)\end{array}$

$16.78(14.48-19.08)$
$103.2(100.00-106.30)$
$0.13(-084$ to 1.09$)$
$3.97(3.18-4.76)^{\S}$
$7.85(2.65-13.05)$
$94.09(84.94-103.20)$
$0.33(0.29-0.37)$
$4.15(3.85-4.46)$
$141.00(88.96-193.00)$
$168.60(152.70-184.50)$
$53.36(45.07-61.66)$
$89.70(79.80-99.60)$
$40.45(35.64-45.27)^{* *}$
$24.18(14.55-33.81)^{*}$
$9.70(8.69-10.71)$

0.2074

0.6612

0.2282

0.0153

0.3685

0,3403

0.4122

0.7919

0.1170

0.0430

0.9132

0.0905

0.0070

0.0464

0.2310

Children are grouped according to the body mass index of their mothers. Fasting length refers to the interval of time between the last meal and the time at which blood samples were collected. Values correspond to the mean \pm SEM. $* p<0.05 ; * * p<0.01 ; * * *<<0.001$ compared with mothers of normal body weight or with children born to mothers of normal body weight; ${ }^{\S \S} p<0.001$ compared with overweight mothers (as determined by one-way ANOVA followed by Tukey's multiple comparison test)

$H D L-C$ high-density lipoprotein cholesterol, $L D L-C$ low-density lipoprotein cholesterol, $A S T$ aspartate aminotransferase, $A L T$ alanine aminotransferase, $G G T$ gammaglutamyl-transferase

signals detected in the same plasma sample. Thereafter, normalized data were submitted to unsupervised "principal component analysis" (PCA), followed by the construction of orthogonal partial least-squares discriminant (OPLS-DA) models using SIMCA-P+ software (Umetrics, Umea, Sweden). For the construction of the S-Plots corresponding to the OPLS-DA analysis, both overweight and obese groups were merged and compared with the normal body weight group. PCA analysis determines the global spectral differences between two or more experimental groups and allows one to establish an initial hypothesis about the variables that drive the differences among the groups. OPLS-DA analysis permits to verify the hypothesis and to get information about the variables in which samples are distinguished. Corresponding OPLS models S-Plots permits a visualization of the most discriminant features between dyslipidemic status or origins. We therefore used PCA to identify the global lipidomic differences between the groups and OPLS-DA to reveal specific lipidomic changes and improve the separation between the different lipid classes. Models validity was appraised using permutation tests and CV-ANOVA. Data related to the clinical characteristics of mothers and offspring and the results of the direct quantification of ceramides, were checked for normality before statistical processing using the D'Agostino \& Pearson omnibus normality test. Data are presented as mean \pm SEM 


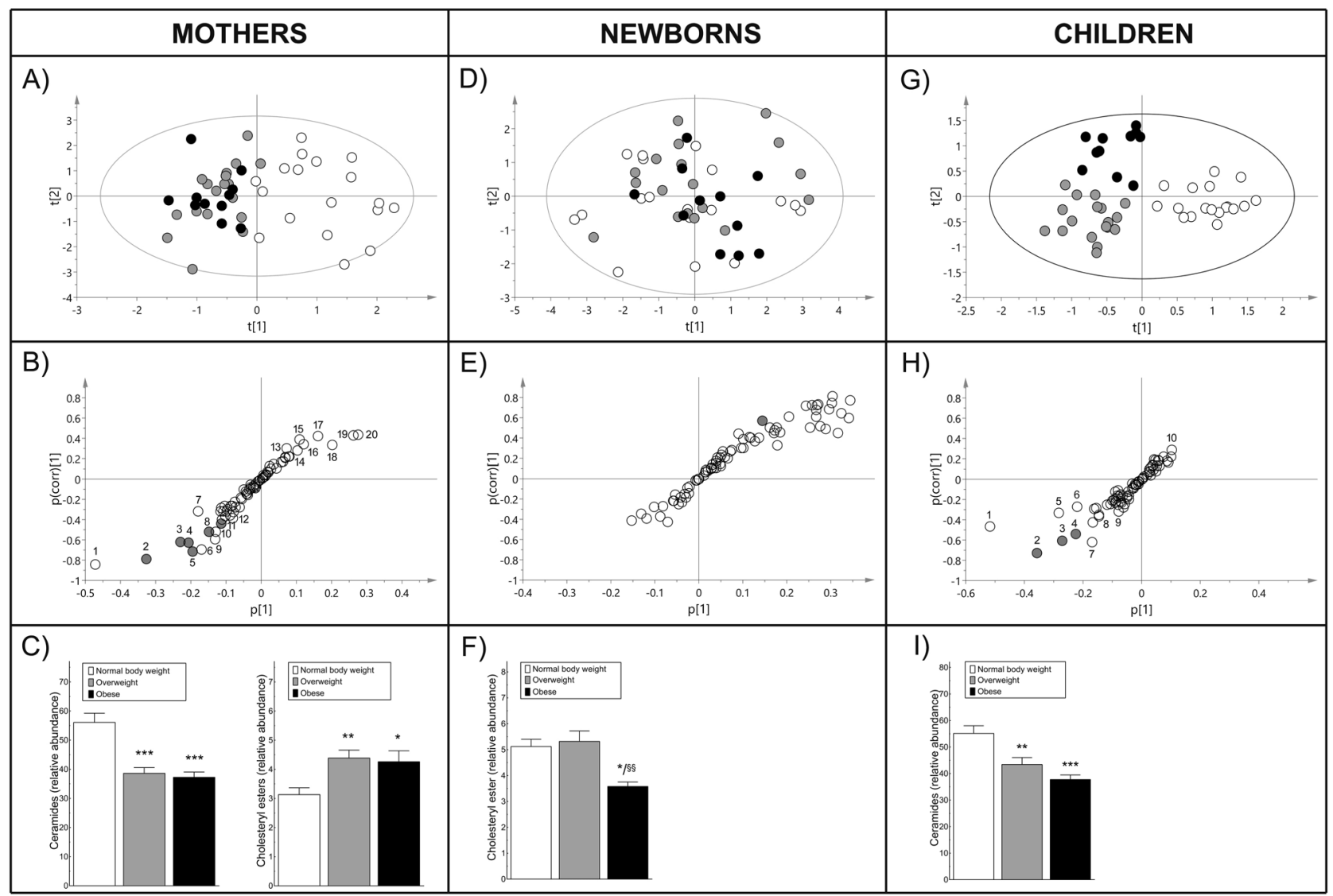

Fig. 1 Global differences in plasma lipidome between mothers and between children of normal body weight (NBW), overweight (OW) or obese (OB) mothers. Scores plots of the OPLS-DA models of lipidomic profile in mothers (a) and their newborns (d) and children (g). Note the clear segregation pattern among the data from the NBW (open circles), OW (gray circles), and OB (black circles) groups of mothers and children but not among the data of newborns. Representative OPLS-DA loading S-plot showing the distribution patterns of specific lipids contributing to the differences between NBW, OW, and OB mothers (b) and between their newborns (e) and children (h) on the score plots. Note that though some lipids show a discriminative pattern in newborns, these differences are not statistically significant as indicated by the results of the multivariate model $\left(R^{2}(X)=0.197, R^{2}\right.$ $(Y)=0.225$, and $Q^{2}(Y)=-0.165, p>0.05$. Gray circles in (b) correspond to the following ceramide species: $2=\operatorname{Cer}(\mathrm{d} 18: 1 / 24: 0) ; 3=$ Cer $(\mathrm{d} 18: 1 / 24: 1) ; 4=\operatorname{Cer}(\mathrm{d} 18: 1 / 23: 0) ; 5=\operatorname{Cer}(\mathrm{d} 18: 1 / 20: 0) ; 8=$ Cer $(\mathrm{d} 18: 1 / 22: 0) ; 11=\operatorname{Cer}(\mathrm{d} 18: 1 / 18: 0)$. Other discriminating lipids are: 1 $=\mathrm{PC}(36: 2) ; 6=\mathrm{SM}(20: 0) ; 7=\mathrm{FA}(18: 3) ; 9=\mathrm{SM}(22: 0) ; 10=\mathrm{LPC}$

(standard error to the mean). Statistical differences between groups were assessed by one-way ANOVA or one-way analysis of covariance (ANCOVA) followed by Tukey's or Dunnett's multiple comparison tests. Correlations were performed using multiple regression analysis, Pearson's correlation coefficient, and Partial correlation. Statistical analyses were performed with GraphPad Prism software (version 6.0, GraphPad Software Inc., La Jolla, CA, USA), and IBM SPSS. Statistical significance was set at $p<0.05$.
$(20: 5) ; 12=\mathrm{SM}(24: 1) ; 13=\mathrm{PC}(36: 6) ; 14=\mathrm{PE}(38: 4) ; 15=\mathrm{DG}$ $(34: 1) ; 16=\mathrm{SM}(16: 0) ; 17=\mathrm{CE}(18: 2) ; 18=\mathrm{FA}(18: 1) ; 19=\mathrm{FA}$ (22:5); $20=$ FA (20:3). Gray circles in (h) correspond to the following ceramide species $2=\operatorname{Cer}(18: 1 / 24: 0) ; 3=\operatorname{Cer}(18: 1 / 23: 0)$; Cer $(18: 1 /$ 22:0). Other discriminating lipids are: $1=\mathrm{FA}(16: 0) ; 5=\mathrm{FA}(16: 1) ; 6$ $=$ FA (20:3); $7=\mathrm{SM}(24: 0) ; 8=\mathrm{PC}(36: 2 \mathrm{e}) ; 9=\mathrm{LPC}(18: 0 \mathrm{e}) ; 10=$ SM (24:1); $11=$ TG (50:3). Relative abundance of total plasma ceramides and cholesteryl esters in mothers (c), of total cholesteryl esters in newborns (f) and of total ceramides in children (i). In all cases, relative abundance was calculated by normalizing the sum of the intensities of all signals of the corresponding lipid to the sum of the intensity signals of all the variables detected in the quality control samples. $* * * p<0.001 ; * * p<0.01 ; * p<0.05$ compared with mothers of normal body weight or with children/newborns of normal body weight mothers. ${ }^{\S} p<0.01$ compared with newborns of normal body weight mothers. All statistical differences were assessed by one-way ANOVA followed by Tukey's multiple comparisons test

\section{Results}

\section{Clinical characteristics of the study population}

The maternal and childhood characteristics of the different groups of patients are presented in Table 1. In spite of the differences in BMI, mothers exhibited the same bodyweight gain during pregnancy. Moreover, the anthropometric characteristics of the children, both at birth and at 4 years, were within normal reference values independently of 
Table 2 . Maternal lipidomic profile in plasma according to body mass index

\begin{tabular}{lrllr}
\hline Type of Lipid & \multicolumn{2}{l}{ Maternal body weight characteristics } & \multirow{2}{*}{ One-way ANOVA $p$-value } \\
\cline { 2 - 4 } & $\begin{array}{l}\text { Normal weight } \\
(n=18)\end{array}$ & $\begin{array}{l}\text { Overweight } \\
(n=18)\end{array}$ & $\begin{array}{l}\text { Obese } \\
(n=11)\end{array}$ & \\
\hline LPC (18:0) & $134.41 \pm 4.02$ & $127.27 \pm 4.35$ & $114.45 \pm 5.31 *$ & 0.0335 \\
SM (d18:1//16:0) & $261.50 \pm 7.80$ & $334.80 \pm 28.70^{*}$ & $264.50 \pm 9.02$ & 0.0227 \\
SM (d18:1/20:0) & $135.70 \pm 3.03$ & $114.67 \pm 3.12 * * *$ & $112.28 \pm 3.92^{* * *}$ & $<0.0001$ \\
Cer (d18:1/18:0) & $1.32 \pm 0.10$ & $0.87 \pm 0.04 * *$ & $0.95 \pm 0.05 *$ & 0.0004 \\
Cer (d18:1/20:0) & $1.96 \pm 0.10$ & $1.27 \pm 0.05^{* * *}$ & $1.35 \pm 0.07 * * *$ & $<0.0001$ \\
Cer (d18:1/22:0) & $9.42 \pm 0.39$ & $7.75 \pm 0.30^{* *}$ & $7.81 \pm 0.31 *$ & 0.0037 \\
Cer (d18:1/23:0) & $6.35 \pm 0.38$ & $4.46 \pm 0.25 * * *$ & $4.56 \pm 0.25 * *$ & 0.0001 \\
Cer (d18:1/24:0) & $20.61 \pm 1.86$ & $11.32 \pm 0.66^{* * *}$ & $11.19 \pm 0.67 * * *$ & $<0.0001$ \\
Cer (d18:1/24:1) & $14.97 \pm 0.95$ & $11.59 \pm 0.93 *$ & $10.00 \pm 0.77 * *$ & 0.0031 \\
PE (38:4) & $5.25 \pm 0.42$ & $6.51 \pm 0.45$ & $7.41 \pm 0.78^{*}$ & 0.0321 \\
CE (18:2) & $1.25 \pm 0.14$ & $2.08 \pm 0.25^{*}$ & $2.09 \pm 0.30^{*}$ & 0.0119 \\
DG (34:1) & $0.72 \pm 0.06$ & $1.00 \pm 0.08^{*}$ & $1.00 \pm 0.11^{*}$ & 0.0061 \\
\hline
\end{tabular}

Data are expressed as mean \pm S.E.M. and correspond to relative abundance of each lipid as determined by normalizing the intensity of its specific signal to the sum of the intensity signals of all the variables detected in the quality control samples. $* p<0.05$; ** $p<0.01$; ***p $<0.001$ compared to the values of mothers of normal body weight. ${ }^{\S} p<0.05$ compared with overweight mothers. All comparisons were made using oneway ANOVA analysis followed by Tukey's multiple comparisons test

LPC lysoglycerophosphatidylcholine SM sphingomyelin, Cer ceramide, $P C$ glycerophosphatidylcholine, $P E$ glycerophosphatidylethanolamine, $C E$ cholesteryl ester, $D G$ diglyceride the BMI of their mothers. However, at 4 years old, the offspring of overweight and obese mothers presented higher plasma concentrations of AST and ALT compared with children of mothers of normal body weight. Nevertheless, the plasma concentrations of these metabolites were below the cut-off reference values of hepatic steatosis. Apart from these differences, the plasma metabolite profile of all children at 4 years was very similar.

\section{Maternal lipidome fingerprints}

The automatic processing of the raw mass spectrometry data indicated the presence of 2356 variables in all plasma samples. From these, 158 lipid markers were identified and selected with the use of our in-house lipidomic database (Supplemental Table S3). Thereafter, all features related to an isotopic overlapping signal were excluded and features corresponding to a single marker detected in several adducts were summed. We took into account only those lipids that were present in all groups and in every patient of each group. As a result of this cleaning process, 68 lipid markers, exhibiting a RSD value below $30 \%$ in QC samples $(n=12)$, were used for analysis (Supplemental Table S4).

As expected, PCA of the data showed that the lipid fingerprints of obese and overweight mothers were clustered closely and separated from lean mother's fingerprints (Supplemental Fig. 2). The first component explained $19.7 \%$ of the variance and the second one $13.1 \%$.
Supervised OPLS-DA statistical models were subsequently performed in order to determine the relative contribution of the various classes of lipids to the differences in the lipidomic profile between the maternal groups and to identify putative biomarkers (Fig. 1a). The performance characteristics associated to the resulting multivariate model were $R^{2}$ $(X)=0.285, R^{2}(Y)=0.719$, and $Q^{2}(Y)=0.420$ ( $p$-value of $\mathrm{CV}-\mathrm{ANOVA}=0.0001)$. Using as discrimination criterion the lipids located in the upper right and lower left squares of the OPLS-DA-associated S-plot (Fig. 1b), we noticed that glycerophosphatidylchonines (PC), ceramides and free fatty acids (FA) were the lipid class contributing the most to the differences between the groups. Quantitative examination of the data followed by one-way ANOVA further showed that these differences were mainly associated with a decrease in the total abundance of $\operatorname{Cer}\left(F_{(2,44)}=17.34, p<0.0001\right)$, along with an increase in cholestery esters $\left(F_{(2,41)}=6.230\right.$, $p=0.043$ ), in obese and overweight mothers compared with mothers of normal body weight (Fig. 1c). This was confirmed by the analysis of the relative abundance of each individual lipid (Table 2). Indeed, the plasma concentrations of six out of the seven species of ceramides that could be identified were decreased in overweight and obese mothers compared with their lean counterparts. Lysoglycerophosphatidylcholine (LPC) 18:0, sphingomyelin (SM) d18:1/ 20:0, glycerophosphatidylethanolamine (PE) 38:4; cholesteryl ester (CE) (18:2), and diglycerides (DG) (34:1) exhibited also a discriminative profile (Table 2). 
Table 3 Impact of maternal obesity on the serum lipidomic profile of the offspring

\begin{tabular}{|c|c|c|c|c|}
\hline \multirow[t]{2}{*}{ Type of Lipid } & \multicolumn{3}{|c|}{ Maternal body weight characteristics } & \multirow[t]{2}{*}{ One-way ANOVA $p$-value } \\
\hline & $\begin{array}{l}\text { Normal weight } \\
(n=18)\end{array}$ & $\begin{array}{l}\text { Overweight } \\
(n=18)\end{array}$ & $\begin{array}{l}\text { Obese } \\
(n=11)\end{array}$ & \\
\hline \multicolumn{5}{|l|}{ Newborns } \\
\hline SM (d18:1/22:0) & $121.52 \pm 2.04$ & $130.79 \pm *$ & $149.28 \pm 3.42 * * * \S \S \S$ & $<0.0001$ \\
\hline Cer (d18:1/18:0) & $0.43 \pm 0.03$ & $0.53 \pm 0.03 *$ & $0.40 \pm 0.02$ & 0.0201 \\
\hline PC (40:4) & $22.43 \pm 1.51$ & $25.32 \pm 1.75$ & $32.39 \pm 1.28 * * * / \S$ & 0.0009 \\
\hline \multicolumn{5}{|l|}{ Children } \\
\hline Cer $(\mathrm{d} 18: 1 / 22: 0)$ & $9.41 \pm 0.52$ & $7.59 \pm 0.44 *$ & $6.79 \pm 0.35^{* *}$ & 0.0020 \\
\hline Cer $(\mathrm{d} 18: 1 / 23: 0)$ & $7.01 \pm 0.47$ & $5.21 \pm 0.32 * *$ & $4.67 \pm 0.25^{* *}$ & 0.0005 \\
\hline Cer $(\mathrm{d} 18: 1 / 24: 0)$ & $24.65 \pm 1.57$ & $15.48 \pm 1.15^{* * *}$ & $14.29 \pm 0.65 * * *$ & $<0.0001$ \\
\hline $\mathrm{PC}(36: 2 \mathrm{e})$ & $18.44 \pm 0.82$ & $15.32 \pm 0.80 *$ & $15.39 \pm 0.94$ & 0.0180 \\
\hline
\end{tabular}

Data are expressed as mean \pm SEM and correspond to relative abundance of each lipid as determined by normalizing the intensity of its specific signal to the sum of the intensity signals of all the variables detected in the quality control samples. $* p<0.05 ; * * p<0.01 ; * * * p<0.001$ compared to the respective values obtained in serum from babies or children born to mothers of normal body weight using one-way ANOVA analysis followed by Tukey's multiple comparisons test

$S M$ sphingomyelin, Cer ceramide, $P C$ glycerophosphatidylcholine, $T G$ triglyceride, $L P C$ lysoglycerophosphatidylcholine, $D G$ diglyceride

\section{Lipidomic fingerprints of the offspring}

PCA analysis of the data showed that there were no differences by maternal BMI status in the clustering of the lipidomic fingerprints or the total abundance of the different classes of lipids among the offspring at birth (Supplemental Fig. 2), and, consequently, the performance characteristics associated to the resulting multivariate model (Fig. 1d, e), were not statistically significant $\left(R^{2}(X)=0.197, R^{2}(Y)=\right.$ 0.225 , and $Q^{2}(Y)=-0.165, p$-value of CV-ANOVA > 0.05). However, newborn babies of obese mothers had lower total concentration of CEs $\left(F_{2,42}=5.296, p=\right.$ 0.0089 , Fig. 1f), together with higher plasma concentrations of SM (d18:1/22:0) and of PC (40:4) when compared to the offspring of normal weight mothers (Table 3). Maternal overweight, but not maternal obesity, was associated with enhanced plasma levels of Cer (d18:1/18:0), in newborn babies (Table 3).

We observed a segregation of the lipidomic profile of children at 4 years by maternal BMI status by PCA analysis with $15.9 \%$ of the variance explained by component 1 and 15.2\% explained by component 2 (Supplemental Fig. 2). As for mothers, ceramides were the lipids that contributed the most to these differences as indicated by the results of the supervised OPLS statistical analysis $\left(R^{2}(X)=0.374, R^{2}(Y)\right.$ $=0.876$, and $Q^{2}(Y)=0.584, p$-value of CV-ANOVA $<$ 0.0001 , Fig. $1 \mathrm{~g}, \mathrm{~h}$ ), and the quantification of the total abundance of each lipid class $\left(F_{(2,44)}=10.39, p=0.0002\right.$, Fig. 1i). Examination of the plasma levels of each individual lipid further showed that in addition to ceramides (d18:1/22:0), (d18:1/23:0), and (d18:1/24:0), children of overweight and obese mothers exhibited differences in the circulating levels of PC (36:2e) compared with children born to mothers of normal body weight (Table 3 ).

\section{Quantification of plasma ceramides}

The concomitant changes in the plasma levels of ceramides exhibited by overweight and obese mothers and their 4year-old children, indicated that ceramides might be a strong marker of the metabolic disease risk induced in the offspring by maternal obesity. To add further support to this hypothesis and validate the lipidomic data, we performed a direct quantification of 10 different ceramides species in all plasma samples using Cer (d18:1/17:0) as internal standard. Data issued from this study were submitted to one-way ANCOVA, using maternal age, and time of delivery as adjusting factors for mothers and fasting length, sex, and zBMI for children. The results of this study confirmed that both maternal overweight and maternal obesity are associated with significant changes in the circulating levels of ceramides in the offspring. Actually, the plasma concentrations of Cer (d18:1/22:0), Cer (d18:1/23:0), and Cer (d18:1/24:0) were all reduced by $20-35 \%$ among overweight and obese mothers and their 4-year-old infants when compared to lean mothers and their children (Fig. 2). Of note, the concentration of Cer (d18:1/20:0) was also decreased in obese and overweight mothers but not in their children. The results of the analysis of covariance model for mother's data were as follows: Cer 20:0 $F_{(2,42)}=6.187, p=$ 0.004; Cer 22:0 $F_{(2,41)}=4.260, p=0.021$; cer 23:0 $F_{(2,40)}$ $=3.339, p=0.0465$; cer 24:0 $F_{(2,42)}=13.10, p<0.0001$. And those for children's data: Cer 22:0 $F_{(2,39)}=3.888, p=$ 0.044; Cer 23:0 $F_{(2,41)}=6.475, p=0.004$; Cer 24:0 $F_{(2,41)}$ 


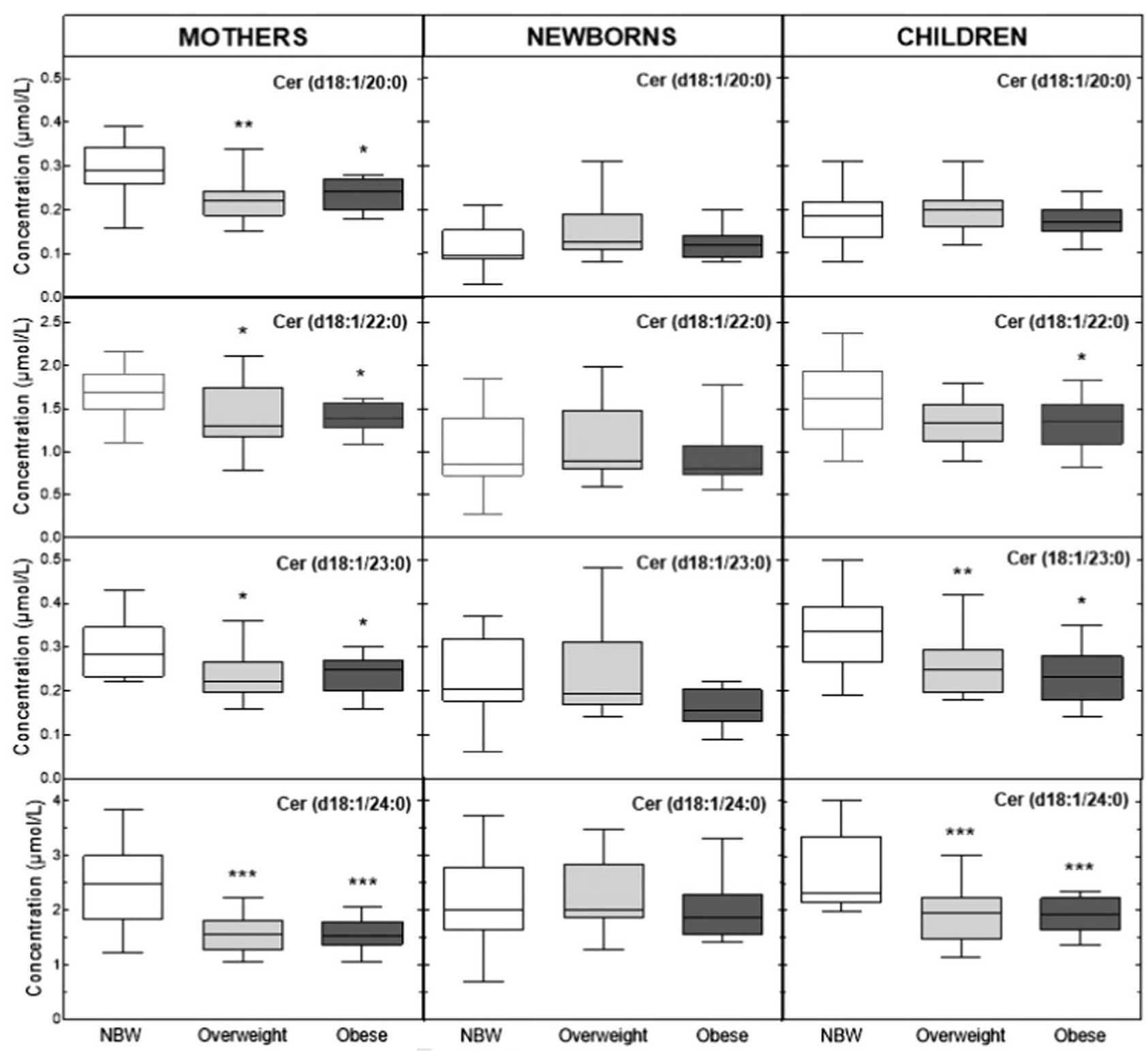

Fig. 2 Plasma concentration of ceramides in mothers and their offspring. Graph represents the boxes and whisker plots corresponding to the concentrations of ceramides in plasma of mothers and their children at birth and at 4years old. Ceramides were quantified by UPLCMS/MS using Cer (18:1/17:0) as internal standard. The boxes represent the 5th and 95th percentiles and horizontal lines within the boxes
$=9.37, p<0.0001$. Partial correlation analysis of the data further showed that maternal plasma concentrations of Cer (d18:1/22:0) and Cer (d18:1/24:0) are also highly correlated with the offspring concentrations at age 4 but not at birth after adjusting for maternal BMI, and maternal age and children fasting length, sex, and zBMI $[r=0.318, p=$ 0.043 for the correlation between maternal and children Cer (d18:1/22:0), and $r=0.554, p<0.0001$ for the correlation between maternal and children Cer (d18:1/24:0)].

To determine whether the BMI of the mother could predict ceramide plasma levels in her 4-year-old children, indicate the medians. $* p<0.05 ; * * p<0.01 ; * * * p<0.001$, versus their corresponding normal body weight counterparts (normal body weight mothers or children of mothers with normal body weight), as determined by one-way ANCOVA followed by Dunnett's multiple comparison test using maternal age, time of delivery, children fasting length, sex, and zBMI as covariates

data were analyzed by multiple regression analysis using a model in which the aforementioned variables were included as confounding factors. As illustrated in Fig. $3 \mathrm{~b}$ and c, there was a statistically significant negative association between maternal BMI and the plasma levels of Cer (d18:1/23:0) $[F$ ${ }_{(5,41)}=2.796, p=0.029, R^{2}=0.254 ; p=0.003$ for maternal $\mathrm{BMI}]$, and the plasma levels of Cer $(\mathrm{d} 18 / 1: 24: 0[F(5,41)=$ 4.399, $p=0.003, R^{2}=0.349 ; p<0.001$ for maternal $\left.\mathrm{BMI}\right]$ in children after adjusting for maternal age, children sex, children zBMI, and children fasting length. We observed also a negative association between maternal BMI and 
Fig. 3 Predictive value of maternal BMI for plasma ceramide concentrations in $4-$ year-old offspring and correlations between disease risk factor associated with obesity and plasma ceramide concentrations in children. Multiple regression analysis of the BMI of mothers versus the plasma concentrations of Cer (d18:1/22:0) (a), Cer (d18:1/ 23:0) (b), and Cer (d18:1/24:0) (c) in their children. Multiple correlation analysis in (a) was adjusted for children fasting length and children zBMI whereas maternal age, children sex, children zBMI, and children fasting length were used as confounding factors for the multiple linear regression analysis illustrated in (b) and (c). Square inserts in the figures correspond to coefficients of determination and the $p$ values of the regressions models. (d), (e) and (c) illustrate Pearson's correlation coefficient analysis of the plasma concentrations of cholesterol versus those of Cer (d18:1/22:0) and Cer (d18:1/ 23:0), and between the circulating levels of AST and Cer (d18:1/22:4) in children. Correlation coefficients $(r)$ and $p$ values are shown
A)

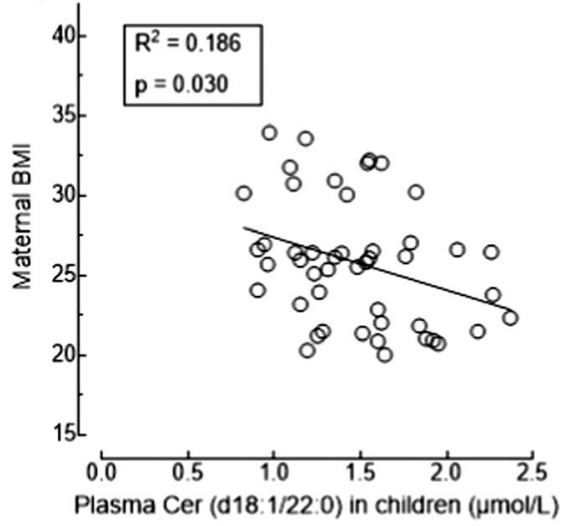

B)

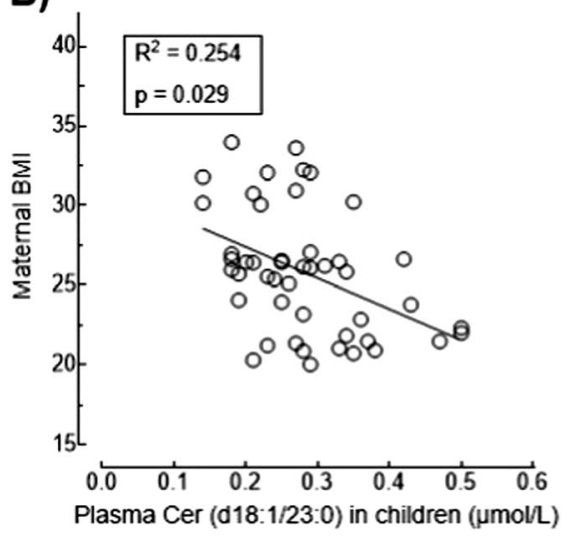

C)

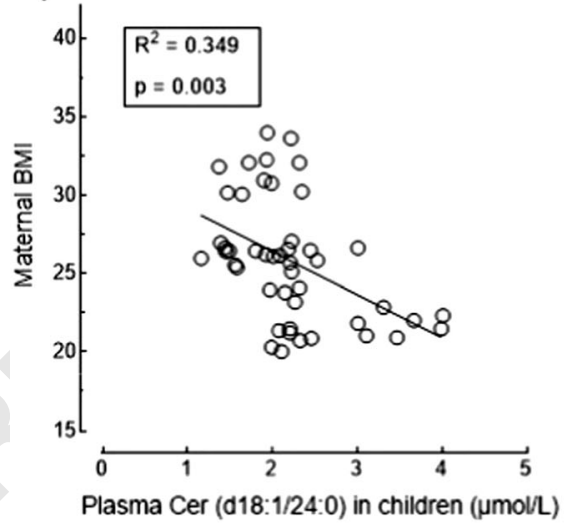

D)

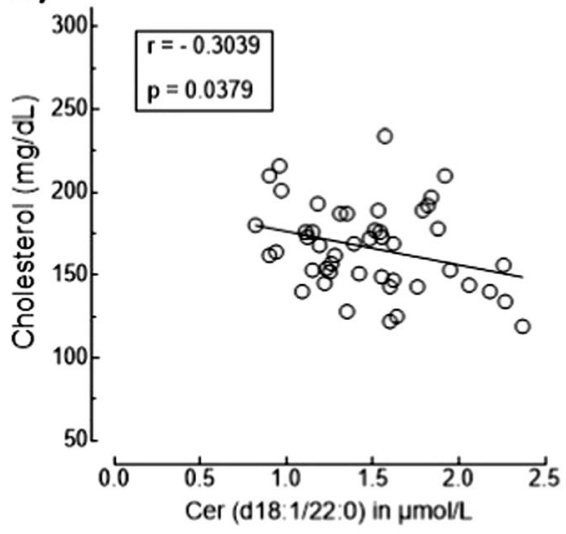

E)

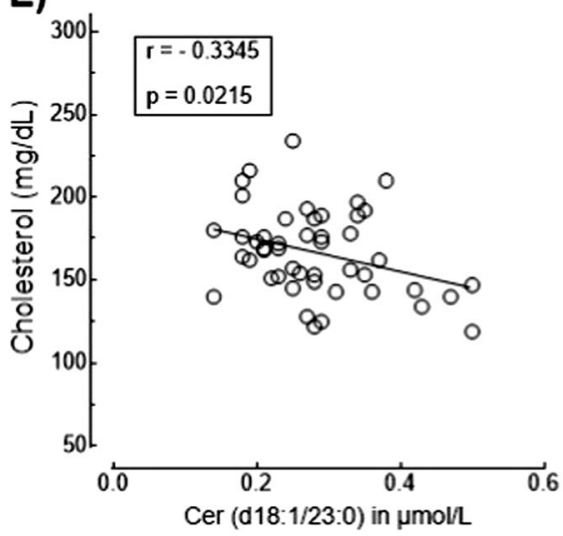

F)

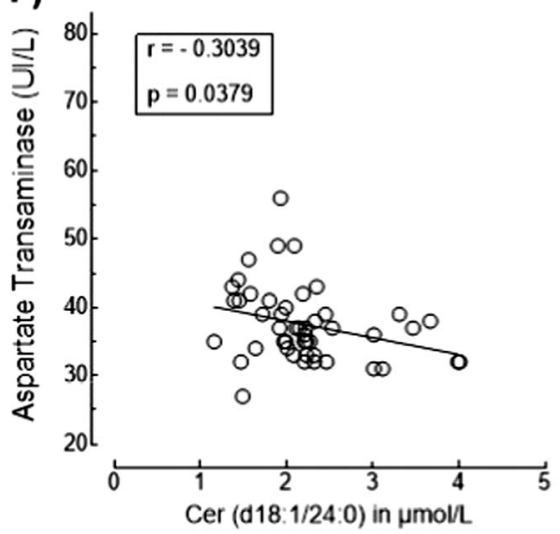

plasma levels of Cer (18:1/22:0), when data were adjusted for children fasting length and children zBMI $[F(3,43)=$ $3.271, p=0.030, R^{2}=0.186 ; p=0.016$ for maternal BMI, Fig. 3a].

We further examined the relationship, in children, between plasma ceramides and other disease risk factors linked to obesity. Using Pearson's correlation analysis, we found a negative association between the levels of cholesterol and those of Cer (d18:1/22:0) and Cer (d18:1/23:0), as well as between the levels of AST and those of Cer (d18:1/
24:0) (Fig. 3d-f). The concentrations of LDL were also negatively correlated with the plasma levels of, respectively, Cer 23:0 and Cer 24:0. Similarly, there was a negative correlation between zBMI and the circulating levels of Cer 22:0 and Cer 24:0. However, none of the associations persisted when the data were submitted to multiple linear regression analysis using sex and fasting length as adjusting variables. This might be explained by the fact that children do not show yet any sign of metabolic 
dysfunction and, therefore, the circulating levels of their metabolites fall within the normal range.

\section{Dietary intake}

To exclude the possibility that the differences in the lipidomic profile between children could result from different familial dietary habits, their dietary intake was evaluated. As shown in Supplemental Table S5, no significant differences in mean daily energy and macronutrient intakes were observed among the different groups of children.

\section{Discussion}

A large number of observational studies indicate that maternal obesity is associated with several adverse health outcomes in offspring including increased BMI and fat mass and enhanced incidence of cardiovascular diseases, type 2 diabetes, and stroke at adulthood. Further studies aimed at identifying the underlying mechanisms of this disease susceptibility, have shown that children of overweight/obese mothers have high circulating levels of triglycerides, insulin, C-peptide, and IL-6 together with low levels of HDL-C and apolipoprotein A-I [15, 16, 28]. Here, we performed a lipidomic analysis and show that obese and overweight pregnant mothers exhibit an altered lipidomic profile in plasma and that the same alterations are present in their offspring at age 4 but not at birth. Specifically, we observed a concomitant decrease in the total relative abundance of ceramides in obese and overweight mothers and their children in comparison to mothers of normal body weight and their offspring. The quantification of several species of ceramides subsequently showed a specific decrease in the concentration of Cer (d18:1/22:0), Cer (d18:1/23:0), and Cer (d18:1/24:0). Interestingly, there were no differences neither in the circulating levels of usual markers of metabolic dysfunction (glucose, insulin, triglycerides, etc.), nor in zBMI among the children of the three groups. Moreover, children exhibited equal daily amounts of energy and food intake independently of the BMI of their mothers. Taken together, these observations suggest that the nutritional status of the mother during pregnancy affects in the long term the lipidomic profile of the children and point out the potential role of ceramides as early predictors of disease risk associated with maternal obesity.

The development of the fetus depends on the provision of nutrients from the maternal circulation. This leads intuitively to think, that there should be a perfect match in the metabolite composition of the maternal and infant plasma at delivery. It may therefore seem paradoxical that there is a correlation between the plasma concentrations of ceramides of mothers and their 4-year-old children, but not between the circulating levels of ceramides of mothers and babies at birth. However, previous studies have consistently shown that the fatty acid composition of umbilical cord plasma is different from that of maternal plasma [29, 30] likely because of the high rate of fatty acid metabolism within the placenta [31].

It has been reported that ceramides are increased in obese humans and are positively related with insulin resistance [32-35]. These results are at odds with the herein presented observations in overweight and obese mothers and raise the question of the physiopathological relevance of the alterations in the concentration of ceramides detected in their children. However, other data from the literature could explain our seemingly contradictory results. First, a close inspection of the clinical phenotype of the patients in which enhanced ceramide levels have been linked with obesity, shows that these patients are already insulin resistant [3235]. In fact, several studies have failed to observe differences in the concentration of ceramides between lean and obese non-diabetic patients [36-39], indicating that an increase in ceramide content is a hall mark of insulin resistance rather than of obesity per se. Second, to date, all the studies linking ceramides to obesity have been performed in biological samples from men or non-pregnant women. This makes it difficult to establish a direct comparison with the present data because of the major adjustments in lipid metabolism occurring during gestation which include dynamic changes in the concentration of ceramides in blood [40]. For instance, it has been shown that the plasmatic levels of glycerophosphatidylcholine (PC), decrease during the second trimester of pregnancy while those of LPC, lysophosphatidylethanolamine (LPE), and lipopolysaccharides (LPS) increase during the same period (Luan et al., 2014). Similarly, plasma levels of several ceramide species have been reported to increase across gestation [40]. Indeed, to the best of our knowledge, this is the first report about the effects of obesity on the circulating levels of ceramides during pregnancy. Third, although the enhanced concentration of ceramides in blood followed by their accumulation in tissues has been proposed to have a key role in obesity-related metabolic disorders, ceramides are also down regulated in several pathological conditions. In particular, a significant decrease in the concentration of ceramides has been reported in the amniotic fluid of women with fetal Down syndrome [41]. Similarly, preeclamptic patients exhibit a reduction of plasma Cer (d18:1/24:0) during the third trimester of pregnancy when compared to match-gestational control women [40]. An inverse relationship between the serum levels of Cer (d18:1/24:1) and the occurrence of ischemic heart disease [42], or the progression of hepatic cirrhosis [43], has also been reported. Finally, in addition to be at high risk of developing metabolic disorders, offspring of obese mothers exhibit also 
adverse neuropsychiatric problems including attention-deficit/hyperactivity disorder (ADHD) [44, 45]. In this respect, it is worth mentioning that children with ADHD have decreased serum levels of ceramides [46]. The herein reported alterations in the concentration of ceramides in children of obese or overweight mothers may therefore constitute an early marker of both cognitive disability and metabolic disease risk.

The de novo ceramide synthesis involves the condensation of serine and palmitoyl-CoA to form 3-ketosphinganine followed by the sequential formation of sphinganine, dihydroceramide, and ceramide. The $\mathrm{N}$-acylation of sphinganine to produce dihydroceramide is catalyzed by a family of six enzymes, the ceramide synthases (CerS), which differ in their specificity toward the acyl CoA chain length used for $\mathrm{N}$-acylation leading to the synthesis of ceramides of different length chain [47, 48]. This is of importance because accumulating evidence indicates that the physiological and pathological effects of ceramides vary according to their acyl chain length [49-51]. In the present study we observed that obese and overweight mothers and their children exhibit decreased circulating levels of ceramides carrying C22:0, C23:0, and C24:0 saturated fatty acyl chains which are produced by ceramide synthase $2[47,52]$. This indicates that maternal obesity is associated with selective changes in ceramide metabolism.

To the best of our knowledge, this is the first report describing the impact of maternal obesity on the offspring lipidome. An additional strength of the study is the simultaneous analysis of the mother-child dyad lipid profile at delivery and its longitudinal nature. Few studies have reported data on children blood metabolites collected from birth into childhood. This approach enabled us to identify potential early markers of disease risk and to get a more comprehensive understanding of the metabolic disturbances associated with maternal obesity that can be transmitted to the offspring. We are nevertheless aware that our study has several limitations that should be addressed in future work. First, our results were obtained on a limited number of patients and, therefore, need to be confirmed in larger cohorts. Second, though children exhibited similar amounts of total calorie intake, we cannot exclude the possibility that the observed lipidomic differences are secondary to genetic factors or to other life style behaviors or socio-economic factors like physical activity, snacking, and family economic income. Finally, all people included in the study were living in the same geographic area. Consequently, it remains to be determined whether the herein reported results can be generalized to other populations.

In conclusion, this study shows that maternal obesity is associated with concomitant changes in the circulating levels of ceramides in mother and their 4-year-old offspring. Given the involvement of ceramides in the pathogenesis of obesity-related pathologies, this finding points out the potential role of ceramides as early predictors of disease risk due to maternal obesity.

Acknowledgements Luis Felipe León Aguilar is the recipient of a doctoral fellowship from the Mexican National Council of Science and Technology (CONACYT). This work was supported by the French National Research Agency (ANR, grant ANR-16-CE21-0007-01), by CONACYT (Grants SALUD-2013-1-202062 and Joint call ANRCONACYT 2015-16-273510), and the Eunice Kennedy Shriver National Institute of Child Health and Human Development, National Institutes of Health (Grant no. R01HD058818). This work was partially supported the ECOS Nord/ANUIES/CONACyT/SEP program (action numbers M12-S01 and 273510). Laurence A. Marchat was supported by COFAA-IPN. We thank the Centre de Recherche en Nutrition Humaine Ouest for the mass spectrometry facilities.

Author contributions LFL-A and MC performed the lipidomic analysis and contributed to data analysis and to the writing of the manuscript; VF-R contributed to data analysis; FH and LAM performed the metabolite determinations in plasma; $A B-V$ designed the data collection instruments, coordinated, and supervised data collection during the age- 4 follow-up and provided the plasma samples; UR and IR conducted the original clinical trial; $\mathrm{MK}$ and $\mathrm{KO}$ supervised the lipidomic analysis and provided direction for data analysis; RM-C provided direction to the original idea; FB-J designed and coordinated the study, analyzed the data, wrote the manuscript and had primary responsibility for the final content. All authors were involved in editing the manuscript and had final approval for the submitted version.

\section{Compliance with ethical standards}

Conflict of interest The authors declare that they have no conflict of interest.

Publisher's note: Springer Nature remains neutral with regard to jurisdictional claims in published maps and institutional affiliations.

\section{References}

1. PR C. Current mapping of obesity. Nutr Hosp. 2013;28(Suppl 5):21-31

2. Organization WH. Obesity and overweight fact sheet. 2017. http://www.who.int/mediacentre/factsheets/fs311/en/.

3. Flegal KM, Carroll MD, Kit BK, Ogden CL. Prevalence of obesity and trends in the distribution of body mass index among us adults, 1999-2010. JAMA. 2012;307:491-7.

4. Huda SS, Brodie LE, Sattar N. Obesity in pregnancy: prevalence and metabolic consequences. Semin Fetal Neonatal Med. 2010;15:70-76.

5. Gavard JA, Artal R. The Association of Gestational Weight Gain with Birth Weight in obese pregnant women by obesity class and diabetic status: a population-based historical cohort study. Matern Child Health J. 2014;18:1038-47.

6. Goldstein RF, Abell SK, Ranasinha S, et al. Association of gestational weight gain with maternal and infant outcomes: a systematic review and meta-analysis. JAMA. 2017;317:2207-25.

7. Segovia SA, Vickers MH, Gray C, Reynolds CM. Maternal obesity, inflammation, and developmental programming. Biomed Res Int. 2014;2014:14.

8. Neri C, Edlow AG. Effects of maternal obesity on fetal programming: molecular approaches. Cold Spring Harbor Perspect Med 2016;6. 
9. Vickers MH. Developmental programming and transgenerational transmission of obesity. Ann Nutr Metab. 2014;64(Suppl. 1):2634.

10. Falk E. Pathogenesis of atherosclerosis. J Am Coll Cardiol. 2006;47(Suppl. 8):C7-C12.

11. Mota M, Banini BA, Cazanave SC, Sanyal AJ. Molecular mechanisms of lipotoxicity and glucotoxicity in nonalcoholic fatty liver disease. Metabolism. 2016;65:1049-61.

12. Engin A. Non-alcoholic fatty liver disease. In: Engin A, Engin A, editors. Obesity and lipotoxicity. Advances in experimental medicine and biology. vol. 960. Cham: Springer; 2017.

13. Di Meo S, Iossa S, Venditti P. Skeletal muscle insulin resistance: role of mitochondria and other ROS sources. J Endocrinol. 2017;233:R15-R42.

14. Kitessa SM, Abeywardena MY. Lipid-induced insulin resistance in skeletal muscle: the chase for the culprit goes from total intramuscular fat to lipid intermediates, and finally to species of lipid intermediates. Nutrients. 2016;8:466.

15. Gaillard R, Steegers EAP, Duijts L, Felix JF, Hofman A, Franco $\mathrm{OH}$, et al. Childhood cardiometabolic outcomes of maternal obesity during pregnancy: the Generation R Study. Hypertension. 2014;63:683-91.

16. Fraser A, Tilling K, Macdonald-Wallis C, Sattar N, Brion M-J, Benfield L, et al. Association of Maternal Weight Gain in Pregnancy With Offspring Obesity and metabolic and vascular traits in childhood. Circulation. 2010;121:2557.

17. Rai S, Bhatnagar S. Novel lipidomic biomarkers in hyperlipidemia and cardiovascular diseases: an integrative biology analysis. OMICS. 2017;21:132-42.

18. Quehenberger O, Dennis EA. The human plasma lipidome. New Engl J Med. 2011;365:1812-23.

19. Ramakrishnan U, Stein AD, Parra-Cabrera S, Wang M, ImhoffKunsch B, Juárez-Márquez $\mathrm{S}$, et al. Effects of docosahexaenoic acid supplementation during pregnancy on gestational age and size at birth: randomized, double-blind, placebo-controlled trial in Mexico. Food Nutr Bull. 2010;31(2_Suppl. 2):S108-S116.

20. Hernández-Avila MRI, Parra S, Hernández-Avila J, Madrigal H, Willett W. Validity and reproducibility of a food frequency questionnaire to assess dietary intake of women living in Mexico City. Salud Publica Mex. 1998;40:133-40.

21. Romieu I, Barraza-Villarreal A, Escamilla-Núñez C, TexcalacSangrador JL, Hernandez-Cadena L, Díaz-Sánchez D, et al. Dietary intake, lung function and airway inflammation in Mexico City school children exposed to air pollutants. Respir Res. 2009;10:122-122.

22. Gutierrez-Gomez Y, Stein AD, Ramakrishnan U, BarrazaVillarreal A, Moreno-Macias H, Aguilar-Salinas C, et al. Prenatal docosahexaenoic acid supplementation does not affect nonfasting serum lipid and glucose concentrations of offspring at 4 years of age in a follow-up of a randomized controlled clinical trial in Mexico. J Nutr. 2017;147:242-7.

23. Croyal M, Kaabia Z, León L, Ramin-Mangata S, Baty T, Fall F et al. Fenofibrate decreases plasma ceramide in type 2 diabetes patients: a novel marker of CVD? Diab Metab 2017.

24. Ferchaud-Roucher V, Croyal M, Moyon T, Zair Y, Krempf M, Ouguerram K. Plasma lipidome analysis by liquid chromatography-high resolution mass spectrometry and ion mobility of hypertriglyceridemic patients on extended-release nicotinic acid: a pilot study. Cardiovasc Drugs Ther. 2017;31:269-79.

25. Bligh EG, Dyer WJ. A rapid method of total lipid extraction and purification. Can J Biochem Physiol. 1959;37:911-7.

26. Gallart-Ayala H, Courant F, Severe S, Antignac JP, Morio F, Abadie J, et al. Versatile lipid profiling by liquid chromatographyhigh resolution mass spectrometry using all ion fragmentation and polarity switching. Preliminary application for serum samples phenotyping related to canine mammary cancer. Anal Chim Acta. 2013;796(Suppl. C):75-83.

27. Sandra K, Pereira AdS, Vanhoenacker G, David F, Sandra P. Comprehensive blood plasma lipidomics by liquid chromatography/quadrupole time-of-flight mass spectrometry. J Chromatogr A. 2010;1217:4087-99.

28. Oostvogels AJJM, Stronks K, Roseboom TJ, van der Post JAM, van Eijsden M, Vrijkotte TGM. Maternal prepregnancy BMI, offspring's early postnatal growth, and metabolic profile at age 56 years: the ABCD study. J Clin Endocrinol Metab. 2014;99:3845-54.

29. Wijendran V, Bendel RB, Couch SC, Philipson EH, Cheruku S, Lammi-Keefe CJ. Fetal erythrocyte phospholipid polyunsaturated fatty acids are altered in pregnancy complicated with gestational diabetes mellitus. Lipids. 2000;35:927-31.

30. Gil-Sánchez A, Larqué E, Demmelmair H, Acien MI, Faber FL, Parrilla JJ, et al. Maternal-fetal in vivo transfer of [13C]docosahexaenoic and other fatty acids across the human placenta $12 \mathrm{~h}$ after maternal oral intake. Am J Clin Nutr. 2010;92:115-22.

31. Perazzolo S, Hirschmugl B, Wadsack C, Desoye G, Lewis RM, Sengers BG. The influence of placental metabolism on fatty acid transfer to the fetus. J Lipid Res. 2017;58:443-54.

32. Blachnio-Zabielska AU, Koutsari C, Tchkonia T, Jensen MD. Sphingolipid content of human adipose tissue: relationship to adiponectin and insulin resistance. Obesity. 2012;20:2341-7.

33. Kolak M, Westerbacka J, Velagapudi VR, Wågsäter D, Yetukuri $\mathrm{L}$, Makkonen J, et al. Adipose tissue inflammation and increased ceramide content characterize subjects with high liver fat content independent of obesity. Diabetes. 2007;56:1960.

34. Haus JM, Kashyap SR, Kasumov T, Zhang R, Kelly KR, DeFronzo RA, et al. Plasma ceramides are elevated in obese subjects with type 2 diabetes and correlate with the severity of insulin resistance. Diabetes. 2009;58:337.

35. Adams JM, Pratipanawatr T, Berria R, Wang E, DeFronzo RA, Sullards MC, et al. Ceramide content is increased in skeletal muscle from obese insulin-resistant humans. Diabetes. 2004:53:25.

36. Bergman BC, Brozinick JT, Strauss A, Bacon S, Kerege A, Bui $\mathrm{HH}$, et al. Serum sphingolipids: relationships to insulin sensitivity and changes with exercise in humans. Am J Physiol Endocrinol Metab. 2015;309:E398.

37. Majumdar I, Mastrandrea LD. Serum sphingolipids and inflammatory mediators in adolescents at risk for metabolic syndrome. Endocrine. 2012;41:442-9.

38. Hanamatsu H, Ohnishi S, Sakai S, Yuyama K, Mitsutake S, Takeda $\mathrm{H}$, et al. Altered levels of serum sphingomyelin and ceramide containing distinct acyl chains in young obese adults. Nutr . 2014;4:e141.

39. Mamtani M, Meikle PJ, Kulkarni H, Weir JM, Barlow CK, Jowett $\mathrm{JB}$, et al. Plasma dihydroceramide species associate with waist circumference in Mexican American families. Obesity. 2014;22:950-6.

40. Dobierzewska A, Soman S, Illanes SE, Morris AJ. Plasma crossgestational sphingolipidomic analyses reveal potential first trimester biomarkers of preeclampsia. PLoS One. 2017;12: e0175118.

41. Charkiewicz K, Blachnio-Zabielska A, Zbucka-Kretowska M, Wolczynski S, Laudanski P. Maternal plasma and amniotic fluid sphingolipids profiling in fetal Down syndrome. PLoS One. 2015; 10:e127732.

42. Argraves KM, Sethi AA, Gazzolo PJ, Wilkerson BA, Remaley AT, Tybjaerg-Hansen A, et al. S1P, dihydro-S1P and C24:1-ceramide levels in the HDL-containing fraction of serum inversely correlate with occurrence of ischemic heart disease. Lipids Health Dis. $2011 ; 10: 70$. 
48. Mullen TD, Hannun YA, Obeid LM. Ceramide synthases at the centre of sphingolipid metabolism and biology. Biochem J. 2012;441:789-802.

49. Hartmann D, Lucks J, Fuchs S, Schiffmann S, Schreiber Y, Ferreirós $\mathrm{N}$, et al. Long chain ceramides and very long chain ceramides have opposite effects on human breast and colon cancer cell growth. Int J Biochem Cell Biol. 2012;44:620-8.

50. Grösch S, Schiffmann S, Geisslinger G. Chain length-specific properties of ceramides. Prog Lipid Res. 2012;51:50-62.

51. Ben-David O, Futerman AH. The role of the ceramide acyl chain length in neurodegeneration: involvement of ceramide synthases. Neuromolecular Med. 2010;12:341-50.

52. Kihara A. Synthesis and degradation pathways, functions, and pathology of ceramides and epidermal acylceramides. Prog Lipid Res. 2016;63:50-69.
46. Henríquez-Henríquez M, Solari S, Quiroga T, Kim B, Deckelbaum R, Worgall T. Low serum sphingolipids in children with attention deficit-hyperactivity disorder. Front Neurosci 2015; 9.

47. Levy M, Futerman AH. Mammalian ceramide synthases. IUBMB Life. 2010;62:347-56. 


\section{Author Query Form}

\section{Please ensure you fill out your response to the queries raised below and return this form along with your corrections}

\section{Dear Author}

During the process of typesetting your article, the following queries have arisen. Please check your typeset proof carefully against the queries listed below and mark the necessary changes either directly on the proof/online grid or in the 'Author's response' area provided below

\begin{tabular}{|c|c|c|}
\hline Queries & Details Required & Author's Response \\
\hline AQ1 & $\begin{array}{l}\text { Please check your article carefully, coordinate with any co-authors and enter all final edits clearly } \\
\text { in the eproof, remembering to save frequently. Once corrections are submitted, we cannot } \\
\text { routinely make further changes to the article. }\end{array}$ & \\
\hline AQ2 & $\begin{array}{l}\text { Note that the eproof should be amended in only one browser window at any one time; otherwise } \\
\text { changes will be overwritten. }\end{array}$ & \\
\hline AQ3 & $\begin{array}{l}\text { Author surnames have been highlighted. Please check these carefully and adjust if the first name } \\
\text { or surname is marked up incorrectly. Note that changes here will affect indexing of your article in } \\
\text { public repositories such as PubMed. Also, carefully check the spelling and numbering of all } \\
\text { author names and affiliations, and the corresponding email address(es). }\end{array}$ & \\
\hline AQ4 & $\begin{array}{l}\text { Please note that after the paper has been formally accepted you can only provide amended } \\
\text { Supplementary Information files for critical changes to the scientific content, not for style. You } \\
\text { should clearly explain what changes have been made if you do resupply any such files. }\end{array}$ & \\
\hline AQ5 & $\begin{array}{l}\text { There is no footnote corresponding to superscript letter ' } \$ \text { ' in Table } 1 \text {. Please provide the footnote } \\
\text { or delete the letter from the table body. }\end{array}$ & \\
\hline AQ6 & $\begin{array}{l}\text { Footnote } \S \text { is not linked to anything in the body of Table } 2 \text {. Please indicate where "§" should be } \\
\text { inserted or delete the footnote. }\end{array}$ & \\
\hline AQ7 & $\begin{array}{l}\text { Luan et al. (2014) is not in the reference list. Please either provide the complete reference details } \\
\text { or delete the citation from text. }\end{array}$ & \\
\hline AQ8 & Please provide the page range for ref. 8 & \\
\hline AQ9 & Please check the page range (the first page no. and end page no. are same). & \\
\hline AQ10 & Please provide the page range or article number for ref. 23 & \\
\hline AQ11 & Please provide the page range for ref. 46 & \\
\hline
\end{tabular}

